\title{
Unification of Gravity and Electromagnetism
}

Mohammed A. El-Lakany

*Correspondence author

Cairo University, Egypt

\author{
Mohammed A. El-Lakany \\ Cairo University \\ Egypt
}

Submitted : 8 Feb 2021 ; Published : 5 Mar 2021

\begin{abstract}
Gravity and electromagnetism are two sides of the same coin, which is the clue of this unification. Gravity and electromagnetism are representing by two mathematical structures, symmetric and antisymmetric respectively. Einstein gravitational field equation is the symmetric mathematical structure. Electrodynamics Lagrangian is three parts, for electromagnetic field, Dirac field and interaction term. The definition of canonical energy momentum tensor was used for each term in Electrodynamics Lagrangian to construct the antisymmetric mathematical structure. Symmetric and antisymmetric gravitational field equations are two sides of the same Lagrangian.
\end{abstract}

Keywords: Gravity; Electromagnetism; General theory of Relativity; Quantum field theory; Nuclear and Particle Physics; Astrophysics and cosmology.

Gravity and electromagnetism are two sides of the same coin

Gravitational objects have spin and angular momentum, spin and angular momentum of gravitational objects are related to basic quantum properties of elementary particles. The angular momentum for the sun is given by $J_{\text {sun }}=\mathrm{M}_{\text {sun }} \omega_{\sigma} \mathrm{R}_{\text {sun }}^{2} \approx 10^{50}$ ergs.s, for solar system is $J_{\text {solsys }}=\mathrm{M}_{\text {solsys }} \omega_{\sigma} \mathrm{R}^{2}{ }_{\text {solsys }} \approx 10^{52}$ ergs.s. In the case of a galaxy the angular momentum is given by $J_{g a l}$ $=\mathrm{M}_{\text {gal }} \omega_{\sigma} \mathrm{R}_{\text {gal }}^{2}$ where $\mathrm{M}_{\text {gal }}=10^{45} \mathrm{~g} ; \mathrm{R}_{\text {gal }}^{2}=10^{47} \mathrm{~cm}^{2} ; \omega_{\sigma}=2 \mathrm{x}$ $10^{-18} \mathrm{HZ}$ and the value of angular momentum is $\mathrm{J}_{\text {gal }} \approx 10^{74} \mathrm{ergs.s}$ .Similarly, for cluster of galaxies, the angular momentum is given by $J_{\text {clust }}=\mathrm{M}_{\text {clust }} \omega_{\sigma} \mathrm{R}_{\text {clust }}^{2} \approx 10^{110} \mathrm{~h}$ in Hubble scale and for the universe $J_{\text {univ }} \approx 10^{120}$ h. Spin density $(\sigma=$ spin/volume $)$ is the same for a wide range, for an electron the spin density is given by $\sigma_{e}=\frac{0.5 \hbar}{\frac{4}{3} \pi r_{e}^{3}} \sim 10^{\circ}$ ergs.s $/ c c$

For proton $\sigma_{\rho} \sim 10^{9} \mathrm{ergs.s} / \mathrm{cc}$ also for the solar system we have $\sigma_{\text {solsys }} \sim 10^{9} \mathrm{ergs.s} / \mathrm{cc}$. For a galaxy $\sigma_{g z l}=\frac{10^{100} \hbar}{\frac{4}{3} \pi R_{g a}{ }^{3}} \sim 10^{9} \mathrm{ergs.s} / c c$

and spin density for Universe $\sigma_{w i v}=\frac{10^{120} \hbar}{\frac{4}{3} \pi R_{H}{ }^{3}} \sim 10^{9}$ ergs.s $/ c c$ [1].

Not only this, but also magnetic fields seem to be everywhere that we can look in the universe [2]. Magnetic fields are observed to be of the order of $10^{13} \mathrm{G}$ in neutron stars, $10^{3} \mathrm{G}$ in solar type stars.

Magnetic fields of order a few $\mu \mathrm{G}$ also have been detected in radio galaxies [3]. Magnetic fields are associated with all gravitational objects and gravitational objects are magnetic dipoles. Electromagnetism not tied only to charged particles, but the planets, stars, galaxies and clusters.

Symmetric and antisymmetric mathematical structures Many scientists, like Weyl, Eddington, Einstein, Infeld, Born and Schrodinger, have pursued unification of gravity and electromagnetism. Weyl initiated this unification, Eddington considered connection as the central concept then decomposed its Ricci tensor to symmetric Ricci tensor $\left(R_{\mu v}\right)$ represents gravity and antisymmetric Ricci tensor $\left(\mathrm{R}_{\mathrm{v \sigma}}\right)$ represent electromagnetism. Infeld and Born followed the path of Eddington then derived the Lagrangian $\mathcal{L}_{G R}=\sqrt{-\operatorname{det}\left(g_{\mu v}+F_{v \sigma}\right)}-\sqrt{-g}$, they considered the asymmetric metric $g_{(\mu v)}=g_{\mu \nu}+F_{v \sigma}$ its symmetric term $g_{\mu v}$ represents gravity and antisymmetric term $\left(F_{v \sigma}\right)$ represent electromagnetism and $\mathrm{g}$ is the determinant of the symmetric metric tensor $g_{\mu v}$ [4]. Schrodinger generalized Eddington Lagrangian to new form containing the cosmological constant $(\Lambda)[5]$. Despite the failure of these previous attempts, they in its entirety refers to something cannot be neglected is that gravity and electromagnetism should be representing by two mathematical structures.

Curvature tensor

Riemann tensor in terms of Christoffel's symbols defined by

$$
\mathrm{R}_{\mu \nu \sigma}^{\delta}=\Gamma_{\mu \sigma}^{\lambda} \Gamma_{\lambda v}^{\delta}-\Gamma_{\mu v}^{\lambda} \Gamma_{\lambda \sigma}^{\delta}+\Gamma_{\mu \sigma, v}^{\delta}-\Gamma_{\mu v, \sigma}^{\delta}
$$

Riemann Christoffel tensor is of rank four, contravariant in $\delta$ and covariant in $\mu, v$, and $\sigma$, and also

$$
\mathrm{R}_{\mu v \sigma}^{\delta}=0
$$

Is the necessary condition for the validity of the special theory of Relativity and for the absence of permanent gravitational field or are the necessary and sufficient condition that the space time is flat [6]. 
Lowering the last index in the Riemann Christoffel tensor with the symmetric metric tensor $\mathrm{R}_{\mu v \sigma \varepsilon}=\mathrm{R}_{\mu \nu \sigma}^{\delta} g_{\delta \varepsilon}$ the lowered tensor is symmetric under Interchanging of the first and last pair of indices and antisymmetric in $\mu, \varepsilon$ and in $\nu, \sigma$.

Symmetric and antisymmetric Ricci tensors can be written as follow

$$
\begin{aligned}
& R_{\mu v}=\mathrm{R}_{\mu \nu \delta}^{\delta}=\Gamma_{\mu \delta}^{\lambda} \Gamma_{\lambda \mathrm{v}}^{\delta}-\Gamma_{\mu \mathrm{v}}^{\lambda} \Gamma_{\lambda \sigma}^{\delta}+\Gamma_{\mu \delta, v}^{\delta}-\Gamma_{\mu v, \delta}^{\delta} \\
& R_{v \sigma}=\mathrm{R}_{\mu \nu \sigma}^{\delta}=\Gamma_{\delta \sigma, v}^{\delta}-\Gamma_{\delta v, \sigma}^{\delta}
\end{aligned}
$$

Symmetric and antisymmetric Ricci tensors give us the opportunity to have symmetric and antisymmetric gravitational field equations.

\section{General theory of Relativity}

General relativity is the modern theory of gravity; General theory of relativity relate gravitational field to the curvature of space-time. Symmetric stress energy tensor $T_{\mu v}$ is the source of gravitational field in general theory of relativity. In the presence of permanent gravitational field, the symmetric gravitational field equation is

$$
R_{\mu v}-\frac{1}{2} R g_{\mu v}+\Lambda g_{\mu \nu}=\frac{8 \pi G}{c^{4}} T_{\mu \nu}
$$

$\mathrm{R}$ is the Ricci scalar and $\mathrm{G}$ is the gravitational constant. Einstein-Hilbert action for gravity is given by $S=\int \mathcal{L}_{G R} d V=\int \frac{c^{4}}{16 \pi G}(R-2 \Lambda) \sqrt{-g} d^{4} x, d V=\sqrt{-g} d^{4} x$ invariant volume element and gravity Lagrangian defined by

$$
\mathcal{L}_{G R}=\frac{c^{4}}{16 \pi G}(R-2 \Lambda)
$$

Gravity Lagrangian is a combination of Ricci scalar and cosmological constant.

Electrodynamics

Electrodynamics Lagrangian is given by

$$
\mathcal{L}_{E D}=-\frac{1}{4} F^{v \sigma} F_{v \sigma}+\bar{\psi}\left(i \gamma_{v} D^{v}-m\right) \psi
$$

$\mathrm{F}^{v \sigma}$ is the electromagnetic field strength tensor, $\mathrm{D}^{v}$ is the gauge contravariant derivative, $\Psi$ matter field $\Psi=\gamma_{0} \Psi^{+}$is their adjoint, $i=\sqrt{-1}$ and $\gamma_{v}$ is the four Dirac matrices with $(v=0,1 \ldots$

$3)$. The electromagnetic field strength tensor $\left(\mathrm{F}^{v \sigma}\right)$ is given by

$$
F^{v \sigma}=\left[\begin{array}{cccc}
0 & -E_{1} & -E_{2} & -E_{3} \\
E_{1} & 0 & -B_{3} & B_{2} \\
E_{2} & B_{3} & 0 & -B_{1} \\
E_{3} & -B_{2} & B_{1} & 0
\end{array}\right]_{\text {And }} \quad F_{\nu \sigma}=\left[\begin{array}{cccc}
0 & E_{1} & E_{2} & E_{3} \\
-E_{1} & 0 & -B_{3} & B_{2} \\
-E_{2} & B_{3} & 0 & -B_{1} \\
-E_{3} & -B_{2} & B_{1} & 0
\end{array}\right]
$$

their lowered index counterpart.

The first term of Electrodynamics Lagrangian for the electromagnetic field is given by

$$
\mathcal{L}^{\text {c.m. }}=-\frac{1}{4} F^{v \sigma} F_{v \sigma}
$$

Canonical energy momentum tensor for electromagnetic field Lagrangian is

$$
\theta_{v \sigma}^{e m . m}=\frac{\partial \mathcal{L}^{c-s .}}{\partial\left(\partial^{v} A^{\mu}\right)} \partial_{\sigma} A^{\mu}-g_{v \sigma} \mathcal{L}^{c .}
$$

Using the identity $\frac{\partial\left(F^{v \sigma} F_{v \sigma}\right)}{\partial\left(\partial^{v} \mathrm{~A}^{\mu}\right)}=4 F_{v \mu}$, we find

$$
\theta_{v \sigma}^{e \cdot m}=-F_{v \mu} F_{\sigma}^{\mu}+\frac{1}{4} g_{v \sigma} F_{z} F^{\delta 2}
$$

Equation (10) is not antisymmetric due to the asymmetric Tensor $\left(-F_{\mu \nu} F^{\mu}{ }_{\sigma}\right)[7]$, for this let's suppose that the asymmetric tensor is the sum of symmetric and antisymmetric tensors as follow

$$
-F_{\nu \mu} F_{\sigma}^{\mu}=\partial^{\sigma} \chi_{\sigma \nu \mu}-F_{\nu \mu} F_{\sigma}^{\mu}-\partial^{\sigma} \chi_{\sigma \nu \mu}
$$

The divergence tensor is arbitrary antisymmetric tensor in their first two indices $\left(\chi_{\sigma v \mu}=-\chi_{v \sigma \mu}\right)$, it is constructed from electromagnetic field strength tensor $\left(F_{v \sigma}\right)$ and electromagnetic vector potential $\left(A_{\mu}\right)$.

Equation (11) in terms of this definition can be rewritten as

$-F_{v \mu} F_{\sigma}^{\mu}=\partial^{\sigma}\left(F_{v \sigma} A_{\mu}\right)-F_{v \mu} F_{\sigma}{ }^{\mu}-\partial^{\sigma}\left(F_{v \sigma} A_{\mu}\right)$

Employing the Maxwell equation, we obtain

$$
-F_{v \mu} F_{\sigma}^{\mu}=-\mathrm{j}_{v} A_{\mu}-F_{v \mu} F_{\sigma}^{\mu}+\mathrm{j}_{v} A_{\mu}
$$

The antisymmetric stress energy tensor for electromagnetic field can be written as

$$
T_{v \sigma}^{e m}=\theta_{v \sigma}^{e m}+\mathrm{j}_{v} A_{\mu}+F_{v \mu} F_{\sigma}^{\mu}
$$

And also can be written in the form

$$
T_{v \sigma}^{e m}=j_{v} A_{\mu}+\frac{1}{4} g_{v \sigma} F_{z \bar{z}} F^{\tilde{e x}}
$$

If we multiplied this equation by $\left(\frac{8 \pi G}{c^{4}}\right)$, we find

$\frac{8 \pi G}{c^{4}} T_{v \sigma}^{e . m .}=\frac{8 \pi G}{c^{4}} j_{v} A_{\mu}+\frac{8 \pi G}{c^{4}} g_{v \sigma}\left[\frac{1}{4} F_{\delta \lambda} F^{\delta \lambda}\right]$

The second term in electrodynamics Lagrangian for Dirac field and given by

$$
\mathcal{L}^{\text {Dirac }}=\bar{\psi}\left(\text { i } \gamma_{v} \partial^{v}-m\right) \psi
$$

The canonical energy momentum tensor defined by

$$
\begin{aligned}
& \theta_{v \sigma}{ }^{\text {Dirac }}=\frac{\partial \mathcal{L}^{D_{v o c}}}{\partial\left(\partial^{v} \psi\right)} \partial_{\sigma} \psi+\frac{\partial \mathcal{L}^{D_{\text {ins }}}}{\partial\left(\partial^{v} \psi^{\dagger}\right)} \partial_{\sigma} \psi^{\dagger}-g_{v \sigma} \mathcal{L}^{a_{\text {irs }}} \\
& \theta_{\nu \sigma}^{\text {Droc }}=\bar{\psi} i \gamma_{\nu} \partial_{\sigma} \psi-g_{\omega} \bar{\psi}\left(i \gamma_{\lambda} \partial^{2}-m\right) \psi
\end{aligned}
$$

The canonical energy momentum tensor that has been presented in this equation is not antisymmetric due to the symmetric term $\left(\bar{\psi} i \gamma_{V} \partial_{\sigma} \psi\right)$.. For this, the antisymmetric stress energy tensor can be written as the canonical energy momentum tensor minus this symmetric term as follow

$$
\begin{aligned}
& T_{\nu \sigma}^{\text {Drac }}=\theta_{\nu \sigma}^{\text {Dira }}-\bar{\psi} i \gamma_{\nu} \partial_{\sigma} \psi \\
& T_{\nu}^{\text {Dirac }}=-g_{\nu \sigma} \bar{\psi}\left(i \gamma_{\lambda} \partial^{\lambda}-m\right) \psi
\end{aligned}
$$

Multiplying equation (21) by $\left(\frac{8 \pi G}{c^{4}}\right)$, we have

$\frac{8 \pi G}{c^{4}} T_{v \sigma}^{\text {Dirac }}=-\frac{8 \pi G}{c^{4}} g_{v \sigma} \bar{\psi}\left(i \gamma_{2} \partial^{2}-m\right) \psi$

Third term is the interaction Lagrangian and given by 
$\mathcal{L}^{\text {int. }}=-e \bar{\psi} \gamma_{v} \psi A^{v}$

The canonical energy momentum tensor is given by

$\theta_{v \sigma}{ }^{\text {irt }}=g_{v \sigma} e \bar{\psi} \gamma_{\lambda} \psi A^{\lambda}$

And antisymmetric stress energy tensor is

$T_{v \sigma}^{\text {int }}=g_{v \sigma} e \bar{\psi} \gamma_{\lambda} \psi A^{2}$

Antisymmetric stress energy tensor for interaction Lagrangian is the same canonical energy momentum tensor, multiplying the previous equation by $\left(\frac{8 \pi G}{c^{4}}\right)$ we find

$\frac{8 \pi G}{c^{4}} T_{v \sigma}{ }^{\text {int }}=\frac{8 \pi G}{c^{4}} g_{v \sigma} e \bar{\psi} \gamma_{2} \psi A^{2}$

If we added eqs. (16), (22) to eq. (26), we have

$\frac{8 \pi G}{c^{4}}\left[T_{v \sigma}{ }^{e m}+T_{v \sigma}{ }^{\text {int }}+T_{v \sigma}{ }^{\text {Dirax }}\right]=\frac{8 \pi G}{c^{4}} j_{v} A_{\mu}$

$+\frac{8 \pi G}{c^{4}} g_{v \sigma}\left[\frac{1}{4} F_{d i} F^{d i}+e \bar{\psi} \gamma_{\lambda} \psi A^{\hat{\lambda}}-\bar{\psi}\left(i \gamma_{\hat{\lambda}} \partial^{\hat{\lambda}}-m\right) \psi\right]$

If gauge contravariant derivative $\left(D^{2}=\partial^{2}+i e A^{2}\right)$ is used in the previous equation, we find

$\frac{8 \pi G}{c^{4}}\left[T_{v \sigma}^{e m}+T_{v \sigma}{ }^{\mathrm{int}}+T_{v \sigma}{ }^{\text {Dir } x}\right]=\frac{8 \pi G}{c^{4}} j_{v} A_{\mu}$

$+\left[\frac{2 \pi G}{c^{4}} F_{\delta \hat{\lambda}} F^{\delta i}\right] g_{v \sigma}-\frac{8 \pi G}{c^{4}} g_{v \sigma}\left[\bar{\psi}\left(i \gamma_{\lambda} D^{\hat{\lambda}}-m\right) \psi\right]$

$\frac{8 \pi G}{c^{4}}\left[T_{v \sigma}{ }^{e m}+T_{v \sigma}{ }^{\text {int }}+T_{v \sigma}{ }^{\text {Dirac }}\right]=\frac{8 \pi G}{c^{4}} j_{v} A_{\mu}$

$+\left[\frac{2 \pi G}{c^{4}} F_{\delta \lambda} F^{\delta \lambda}\right] g_{v \sigma}-\frac{1}{2} g_{v \sigma}\left[\frac{16 \pi G}{c^{4}} \bar{\psi}\left(i \gamma_{\lambda} D^{i}-m\right) \psi\right]$

$\frac{8 \pi G}{c^{4}} T_{v \sigma}=R_{v \sigma}+\Lambda g_{v \sigma}-\frac{1}{2} R g_{v \sigma}$

Antisymmetric gravitational field equation is gauge invariant and antisymmetric stress energy tensor can be written in the form

$T_{v \sigma}=\left[T_{v \sigma}{ }^{\text {Divax }}+T_{v \sigma}{ }^{\text {int }}+T_{v \sigma}{ }^{e \cdot m}\right]$

Ricci scalar is proportional to the sum of Dirac and interaction Lagrangians as follow

$R=\frac{16 \pi G}{c^{4}} \bar{\psi}\left(i \gamma_{2} D^{2}-m\right) \psi$

Cosmological constant is a construction from electromagnetic field strength tensor and given by

$\Lambda=\frac{2 \pi G}{c^{4}} F^{w} F_{z}$

Antisymmeric Ricci tensor is given by

$$
R_{v \sigma}=\frac{8 \pi G}{c^{4}} j_{v} A_{\mu}
$$

Antisymmetric Ricci tensor is the antisymmetric term of eq.

(13) multiplied by $\left(\frac{8 \pi G}{c^{4}}\right)$ Substituting by eqs. (32), (33) into eq (6), we have

$$
\begin{aligned}
& \mathcal{L}_{G R}=\frac{c^{4}}{16 \pi G}\left[\frac{16 \pi G}{c^{4}} \bar{\psi}\left(i \quad \gamma_{\lambda} D^{\lambda}-m\right) \psi\right]-\frac{c^{4}}{8 \pi G}\left[\frac{2 \pi G}{c^{4}} F^{\delta \alpha} F_{\delta \alpha}\right] \\
= & \bar{\psi}\left(i \gamma_{\lambda} D^{\lambda}-m\right) \psi-\frac{1}{4} F^{\delta \lambda} F_{\delta \alpha}=\mathcal{L}_{E D}
\end{aligned}
$$

Gravity Lagrangian equal to electrodynamics Lagrangian, but in terms of the second set of indices. Electrodynamics Lagrangian and Its parts can be written in terms of one of two sets of indices, first set is $\{\mu, \nu, \sigma\}$ and second set is $\{\varepsilon, \delta, \lambda\}$

If we multiplied eq. (13) by $\left(\frac{8 \pi G}{c^{4}}\right)$, we find

$-\frac{8 \pi G}{c^{4}} F_{v \mu} F_{\sigma}{ }^{\mu}=R_{\mu v}+R_{v \sigma}$

$R_{\mu \nu}=\frac{8 \pi G}{c^{4}}\left[-\mathrm{j}_{v} A_{\mu}-F_{v \mu} F_{\sigma}{ }^{\mu}\right]$

The symmetric Ricci tensor is the symmetric term of eq. (13) multiplied by $\left(\frac{8 \pi G}{c^{4}}\right)$ and it is the sum of divergence tensor and the asymmetric Ricci tensor. If we substituted by eq. (37) into eq. (3), we find

$-\frac{8 \pi G}{c^{4}} F_{v \mu} F_{\sigma}{ }^{\mu}-\frac{8 \pi G}{c^{4}} \mathrm{j}_{v} A_{\mu}=\Gamma_{\mu \omega^{2}}^{{ }^{\delta}}{ }_{2 v}-\Gamma^{2}{ }_{\mu \nu} \Gamma^{\delta}{ }_{2 \delta}+\Gamma^{\delta}{ }_{\mu \nu, v}, \Gamma^{\delta}{ }_{\mu \nu, \delta}$

Substituting by eq. (34) into eq. (4), we find

$\frac{8 \pi G}{c^{4}} j_{v} A_{\mu}=\Gamma_{\delta, v}^{\delta}-\Gamma_{\delta, \sigma}^{\delta}$

This tensor takes the form of curl of vector as follow

$\frac{8 \pi G}{c^{4}} j_{v} A_{\mu}=\partial_{\nu} \partial_{\sigma} \log \sqrt{-g}-\partial_{\sigma} \partial_{v} \log \sqrt{-g}$

Equation (38) can be divided into two equations as follow

$$
\begin{aligned}
& -\frac{8 \pi G}{c^{4}} F_{\nu \mu} F_{\sigma}{ }^{\mu}=\Gamma^{2}{ }_{\mu \nu} \Gamma^{\delta}{ }_{2 \nu}-\Gamma_{\mu \nu}^{2} \Gamma^{\delta}{ }_{2 \delta} \\
& -\frac{8 \pi G}{c^{4}} j_{\nu} A_{\mu}=\Gamma_{\mu, \delta}^{\delta}-\Gamma_{\mu \nu, \delta}^{\delta}
\end{aligned}
$$

Equation (42) can be rewritten as

$$
\begin{aligned}
& -\frac{8 \pi G}{c^{4}} j_{v} A_{\mu}=\partial_{\nu} \partial_{\mu} \log \sqrt{-g}-\partial_{\delta} \Gamma^{\delta}{ }_{\mu \nu} \\
& \partial_{\nu} \partial_{\mu} \log \sqrt{-g}+\frac{8 \pi G}{c^{4}} j_{\nu} A_{\mu}=\partial_{\delta} \Gamma^{\delta}{ }_{\mu \nu}
\end{aligned}
$$

$\Gamma_{\mu \nu}^{\delta}=\frac{1}{2} g^{\delta \sigma}\left(\partial_{\nu} g_{\sigma \mu}+\partial_{\mu} g_{\sigma v}-\partial_{\sigma} g_{\mu \nu}\right)=\frac{1}{2} g^{\delta \sigma}\left(\partial_{\mu} g_{\sigma v}-\partial_{\sigma} g_{\mu \nu}\right)$

$g_{\mu \sigma}=g_{\sigma \mu}=g_{\sigma}{ }^{\mu}=g_{\mu}{ }^{\sigma}=g_{\mu \nu} g^{1 \sigma}=0$

$g_{\varepsilon \lambda}=g_{\lambda \varepsilon}=g_{\lambda}{ }^{\varepsilon}=g_{\varepsilon}{ }^{\lambda}=g_{\varepsilon \delta} g^{\delta \lambda}=0$

$\partial_{\delta} \Gamma_{\mu \nu}^{\delta}=\frac{1}{2} \partial_{\delta} g^{\delta \sigma} \partial_{\mu} g_{\sigma \nu}-\frac{1}{2} \partial_{\delta} g^{\delta \sigma} \partial_{\sigma} g_{\mu \nu}=$

$\frac{1}{2} \partial_{\delta} \partial_{\mu} g^{\delta}{ }_{\nu}-\frac{1}{2} \partial_{\delta} \partial_{\sigma} g^{\delta \sigma} g_{\mu \nu}$

Substitute by eq. (48) into eq. (44), we find

$\partial_{\nu} \partial_{\mu} \log \sqrt{-g}+\frac{8 \pi G}{c^{4}} j_{\nu} A_{\mu}=\frac{1}{2} \partial_{\delta} \partial_{\mu} g^{\delta}{ }_{\nu}-\frac{1}{2} \partial_{\partial} \partial_{\sigma} g^{\delta \sigma} g_{\mu \nu}$ 
Equating the first term by the first term in this equation, we find

$\partial_{\nu} \partial_{\mu} \log \sqrt{-g}=\frac{1}{2} \partial_{\partial} \partial_{\mu} g^{\delta}{ }_{\nu}$

$\partial_{v} \log \sqrt{-g}=\frac{1}{2} \partial_{\delta} g^{\delta}$

In eq. (49) if we equate the second term by the second term, we find

$\frac{8 \pi G}{c^{4}} j_{v} A_{\mu}=-\frac{1}{2} \partial_{\delta} \partial_{\sigma} g^{\delta \sigma} g_{\mu \nu}$

Equating eq. (52) with eq. (40), we find

$$
\partial_{\nu} \partial_{\sigma} \log \sqrt{-g}-\partial_{\sigma} \partial_{\nu} \log \sqrt{-g}=-\frac{1}{2} \partial_{\partial} \partial_{\sigma} g^{\delta \sigma} g u
$$

Equation (41) can be rewritten in the form

$$
\begin{aligned}
& -\frac{8 \pi G}{c^{4}} F_{\nu \mu} F_{\sigma}{ }^{\mu}=\Gamma^{2}{ }_{\mu \omega^{*}} \Gamma^{\delta}{ }_{2 v}-\Gamma^{2}{ }_{\mu \nu} \partial_{2} \log \sqrt{-g} \\
& \Gamma_{\mu \delta^{\hat{\lambda}}}^{\hat{\delta}} \Gamma_{\lambda \nu}^{\delta}=\left[\frac{1}{2} g^{\lambda \sigma}\left(\partial_{\mu} g_{\sigma \delta}-\partial_{\sigma} g_{\mu \delta}\right)\right]\left[\frac{1}{2} g^{\delta \sigma}\left(\partial_{\nu} g_{\sigma \lambda}+\partial_{\hat{\lambda}} g_{\sigma V}-\partial_{\sigma} g_{\hat{\lambda}}\right)\right] \\
& =\frac{1}{4}\left[g^{2 \sigma} \partial_{\mu} g_{\sigma \delta}-g^{2 \sigma} \partial_{\sigma} g_{\mu \nu}\right]\left[g^{\delta \sigma} \partial_{\nu} g_{\sigma L}+g^{\delta \sigma} \partial_{\nu} g_{\sigma v}-g^{\delta \sigma} \partial_{\sigma} g_{\nu v}\right] \\
& =\frac{1}{4}\left[g^{2 \sigma} \partial_{\mu} g_{\sigma \delta} g^{\delta \sigma} \partial_{\nu} g_{\sigma L}+g^{2 \sigma} \partial_{\mu} g_{\sigma \delta} g^{\delta \sigma} \partial_{2} g_{\sigma \nu}-g^{2 \sigma} \partial_{\mu} g_{\sigma \delta} g^{\delta \sigma} \partial_{\sigma} g_{\lambda v}\right] \\
& =\frac{1}{4}\left[g^{2 \sigma} \partial_{\mu} g_{\sigma}{ }^{\sigma} \partial_{\nu} g_{\sigma L}+g^{2 \sigma} \partial_{\mu} g_{\sigma}{ }^{\sigma} \partial_{\nu} g_{\sigma \nu}-g^{2 \sigma} \partial_{\mu} g_{\sigma}{ }^{\sigma} \partial_{\sigma} g_{\nu \nu}\right] \\
& =\frac{1}{4}\left[\partial_{\mu} g^{2 \sigma} g_{\sigma}{ }^{\sigma} \partial_{\nu} g_{\sigma L}+\partial_{\mu} g^{2 \sigma} g_{\sigma}{ }^{\sigma} \partial_{2} g_{\sigma \nu}-\partial_{\mu} g^{2 \sigma} g_{\sigma}{ }^{\sigma} \partial_{\sigma} g_{\nu \nu}\right] \\
& =\frac{1}{4}\left[\partial_{\mu} g^{2 \sigma} \partial_{\nu} g_{\sigma L}+\partial_{\mu} g^{2 \sigma} \partial_{\lambda} g_{\sigma \nu}-\partial_{\mu} g^{2 \sigma} \partial_{\sigma} g_{\nu v}\right] \\
& =\frac{1}{4}\left[\partial_{\mu} \partial_{\nu} g^{2 \sigma} g_{\sigma \lambda}+\partial_{\mu} \partial_{\lambda} g^{2 \sigma} g_{\sigma \nu}-\partial_{\mu} \partial_{\sigma} g^{2 \sigma} g_{\lambda v}\right] \\
& =\frac{1}{4}\left[\partial_{\mu} \partial_{\nu} g^{2}{ }_{2}+\partial_{\mu} \partial_{2} g_{\nu}^{2}-\partial_{\mu} \partial_{\sigma} g^{\sigma \lambda} g_{\nu \nu}\right] \\
& =\frac{1}{4}\left[\partial_{\mu} \partial_{v} g^{2}{ }_{2}+\partial_{\mu} \partial_{2} g^{2}{ }_{\nu}-\partial_{\mu} \partial_{\sigma} g^{\sigma}{ }_{\nu}\right] \text {. } \\
& \Gamma^{2}{ }_{\mu \omega^{5}} \Gamma^{\delta}{ }_{2 v}=\frac{1}{4}\left[\partial_{\mu} \partial_{\nu} g^{2}{ }_{2}+2 \partial_{\mu} \partial_{\nu} \log \sqrt{-g}-2 \partial_{\mu} \partial_{\nu} \log \sqrt{-g}\right] \\
& =\frac{1}{4} \partial_{\mu} \partial_{v} g_{2}^{2}
\end{aligned}
$$

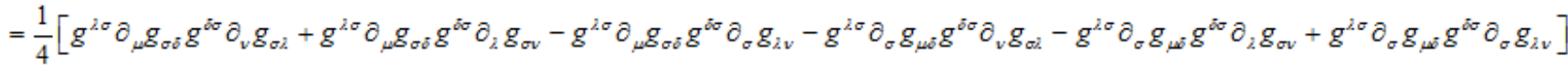

And Substituting by this equation into eq. (54), we have $-\frac{8 \pi G}{c^{4}} F_{v \mu} F_{\sigma}{ }^{\mu}=\frac{1}{4} \partial_{\mu} \partial_{v} g_{2}^{2}+\frac{1}{2} g^{2 \sigma} \partial_{\mu} \partial_{2} \log \sqrt{-g} g_{v \sigma}+\frac{1}{2} g^{2 \sigma} \partial_{\sigma} \partial_{2} \log \sqrt{-g} g_{\mu v}$

Now, let's construct the antisymmetric metric tensor. Electric field in empty space is given by

$$
\vec{E}=\vec{E}_{01} e^{i\left(k_{1} x_{1}-\omega_{1} x_{4}\right)}+\vec{E}_{02} e^{i\left(k_{2} x_{2}-\omega_{2} x_{4}\right)}+\vec{E}_{03} e^{i\left(k_{3} x_{3}-\omega_{3} x_{4}\right)}
$$

$\omega=\omega_{1}+\omega_{2}+\omega_{3}, \vec{k}=\left(k_{1}, k_{2}, k_{3}\right)$ are the wave frequency and wave vector. In general orthogonal curvilinear coordinates a vector A defined as follow

$$
\vec{A}=\vec{e}_{1} h_{1}+\vec{e}_{2} h_{2}+\vec{e}_{3} h_{3} \text {. }
$$

Let's suppose that $\left(\mathrm{E}_{01}, \mathrm{E}_{02}, \mathrm{E}_{\mathrm{n}}\right)$ is the unit vector then equate eq (61) with eq (62), we find $h_{1}=e^{i\left(h_{4} x_{1}-a_{1} x_{4}\right)}, h_{2}=e^{i\left(h_{2} x_{2}-\omega_{2} x_{4}\right)}$ and $h_{3}=e^{i\left(l_{3} x_{3}-\omega_{3} x_{4}\right)} ;$ Using these three coefficients to construct the antisymmetric metric tensor $\left(g_{v \sigma}\right)$, this tensor is in the same

$$
\begin{aligned}
& \Gamma_{\mu \omega^{2}}^{2}=\frac{1}{2} g^{2 \sigma}\left(\partial_{\delta} g_{\sigma \mu}+\partial_{\mu} g_{\sigma \delta}-\partial_{\sigma} g_{\mu \delta}\right)=\frac{1}{2} g^{2 \sigma}\left(\partial_{\mu} g_{\sigma \delta}-\partial_{\sigma} g_{\mu \nu}\right) \\
& \Gamma_{2 v}^{\tilde{\delta}}=\frac{1}{2} g^{\delta \sigma}\left(\partial_{v} g_{\sigma L}+\partial_{2} g_{\sigma v}-\partial_{\sigma} g_{\nu v}\right) \\
& \Gamma_{\mu \nu}^{2}=\frac{1}{2} g^{2 \sigma}\left(\partial_{v} g_{\sigma \mu}+\partial_{\mu} g_{\sigma v}-\partial_{\sigma} g_{\mu v}\right)=\frac{1}{2} g^{2 \sigma}\left(\partial_{\mu} g_{\sigma v}-\partial_{\sigma} g_{\mu v}\right) \\
& =-\frac{1}{2} g^{2 \sigma} \partial_{\mu} g_{v \sigma}-\frac{1}{2} g^{2 \sigma} \partial_{\sigma} g_{\mu \nu}
\end{aligned}
$$

form of electromagnetic field strength tensor $F_{v \sigma}$ and with the same signs.

$$
\begin{aligned}
& g_{v}=\left[\begin{array}{cccc}
0 & h_{1} & h_{2} & h_{3} \\
-h_{1} & 0 & -h_{3} & h_{2} \\
-h_{2} & h_{3} & 0 & -h_{1} \\
-h_{3} & -h_{2} & h_{1} & 0
\end{array}\right]= \\
& {\left[\begin{array}{cccc}
0 & e^{i\left(k_{1} x_{4}-\omega_{1} x_{4}\right)} & e^{i\left(k_{2} x_{2}-\omega_{2} x_{4}\right)} & e^{i\left(k_{3} x_{3}-\omega_{3} x_{4}\right)} \\
-e^{i\left(k_{1} x_{1}-\omega_{1} x_{4}\right)} & 0 & -e^{i\left(k_{3} x_{3}-\omega_{3} x_{4}\right)} & e^{i\left(k_{2} x_{2}-\omega_{1} x_{4}\right)} \\
-e^{i\left(k_{2} x_{2}-\omega_{1} x_{4}\right)} & e^{i\left(k_{3} x_{4}-\omega_{3} x_{4}\right)} & 0 & -e^{i\left(k_{4} x_{4}-\omega_{3} x_{4}\right)} \\
-e^{i\left(k_{3} x_{3}-\omega_{3} x_{4}\right)} & -e^{i\left(k_{2} x_{2}-\omega_{1} x_{4}\right)} & e^{i\left(k_{1} x_{4}-\omega_{1} x_{4}\right)} & 0
\end{array}\right]}
\end{aligned}
$$

And now, we will return to the cosmological constant which it will splits up into two parts where $F_{\delta \lambda} F^{\delta \lambda}=-2 E^{2}+2 B^{2}$

$\Lambda=-\frac{4 \pi G}{c^{4}} E^{2}+\frac{4 \pi G}{c^{4}} B^{2}$

The first term of cosmological constant can be written as $\Lambda_{1}=\frac{8 \pi G}{c^{4}} \rho_{1}$ 


$$
\rho_{1}=-\frac{1}{2} E^{2}
$$

First term is proportional to density of vacuum electric energy, the second term of cosmological constant can be written as

$$
\begin{aligned}
& \Lambda_{2}=\frac{8 \pi G}{c^{4}} \rho_{2} \\
& \rho_{2}=\frac{1}{2} B^{2}
\end{aligned}
$$

Second term is proportional to density of vacuum magnetic energy. Cosmological constant proportional to the sum of binding energy per nucleon
and can be written as $\left(\frac{B \cdot E}{A}\right)$, and its absolute value $\left|\frac{B \cdot E}{A}\right|$,

$\Lambda=\frac{8 \pi G}{c^{4}}\left(\frac{B \cdot E .}{A}\right)+\frac{8 \pi G}{c^{4}}\left|\frac{B \cdot E}{A}\right|$

Binding energy per nucleon is defined by $\frac{B \cdot E}{A}=-\frac{\Delta m}{A} c^{2}$, where $\Delta m=Z m_{p}+(\mathrm{A}-\mathrm{Z}) m_{\mathrm{n}}-\mathrm{M}_{\mathrm{N}}, \mathrm{A}$ is atomic mass number, $\mathrm{Z}$ is atomic number, $\mathrm{M}_{\mathrm{N}}$ is a nucleus mass, $m_{p}$ is proton mass and $m_{n}$ is neutron mass [8].

Symmetric gravitational field equation in empty space is

$R_{\mu \nu}-\frac{1}{2} R g_{\mu \nu}=-g_{\mu \nu} \Lambda$

$R_{\mu \nu}=\left(\frac{1}{2} R-\Lambda\right) g_{\mu v}$

Equating eq. (37) with eq. (71), we find

$-\frac{8 \pi G}{c^{4}} \mathrm{j}_{v} A_{\mu}-\frac{8 \pi G}{c^{4}} F_{v, \mu} F_{\sigma}{ }^{\mu}=\left(\frac{1}{2} R-\Lambda\right) g_{\mu v}$

Antisymmetric gravitational field equation in empty space by analogy to symmetric gravitational field equation is

$$
\begin{aligned}
& R_{v \sigma}-\frac{1}{2} R g_{v \sigma}=-g_{v \sigma} \Lambda \\
& R_{v \sigma}=\left(\frac{1}{2} R-\Lambda\right) g_{v \sigma}
\end{aligned}
$$

Equating eq. (74) with eq. (34), we have

$$
\frac{8 \pi G}{c^{4}} j_{v} A_{\mu}=\left(\frac{1}{2} R-\Lambda\right) g_{v \sigma}
$$

If we added eq. (72) into eq. (75) we have

$$
-\frac{8 \pi G}{c^{4}} F_{v \mu \nu} F_{\sigma}{ }^{\mu}=\left(\frac{1}{2} R-\Lambda\right) g_{v \sigma}+\left(\frac{1}{2} R-\Lambda\right) g_{\mu v}
$$

If we equate eq. (60) by eq. (76), the first term of eq. (60) hasn't comparable one in eq. (76) and equal to zero

$$
\frac{1}{4} \partial_{\mu} \partial_{v} g_{2}^{2}=0
$$

Equating second term of eq. (60) by the first term of eq. (76), we find

$$
\frac{1}{2} g^{2 \sigma} \partial_{\mu} \partial_{2} \log \sqrt{-g}=\frac{1}{2} R-\Lambda
$$

Equating third term of eq. (60) by second term of eq. (76), we find

$$
\frac{1}{2} g^{\lambda \sigma} \partial_{\sigma} \partial_{\lambda} \log \sqrt{-g}=\frac{1}{2} R-\Lambda
$$

Equating eq. (78) by eq. (79), we find

$$
\begin{aligned}
& \frac{1}{2} g^{2 \sigma} \partial_{\sigma} \partial_{2} \log \sqrt{-g}=\frac{1}{2} g^{2 \sigma} \partial_{\mu} \partial_{2} \log \sqrt{-g} \\
& \partial_{\sigma}=\partial_{\mu}
\end{aligned}
$$

\section{Conclusion}

General relativity is very successful theory. This paper introduced new definitions for the cosmological constant, which lead to new study in cosmology undertaken. Differential geometry has been extended by new tensors and operators, these tensors are $g_{\mu \nu}, g_{v \sigma} g_{\varepsilon \delta} g_{\delta \lambda}$. The four dimensional gradient operator became six operators, these operators are $\partial_{\mu}, \partial_{\nu} \partial_{\sigma}, \partial_{\varepsilon}, \partial_{\delta}, \partial_{\lambda}$. This study introduced new relations in differential geometry and created new differential geometry analysis undertaken.

\section{References}

1. Sivaram, C., and Arun, K (2012) "Primordial Rotation of the Universe, Hydrodynamics, Vortices and Angular Momenta of Celestial Objects." The Open Astronomy Journal 5: 7-11.

2. Maartens, R (2000)“Cosmological Magnetic Fields." Pramana Journal of Physics 55(4): 575-583.

3. Kunze, K. E (2009) "Cosmological Magnetic Fields." Journal of Physics; Conference Series 189:12-22. doi:10.1088/1742-6596/189/1/012022.

4. Goenner, H. F. M (2014) "On the History of Unified Field Theories: Part 2.” Living Rev. Relativity 17(1): 5.

5. Poplawski, N. J (2007) "On the Nonsymmetric Purely Affine Gravity.” Modern Physics A 22(36): 2701-2720.

6. Ray, M (1965) "Theory of Relativity: Special and General." Philosophy, Delhi, S. Chand.

7. Greiner, W. Field Quantization. Berlin, Heidelberg, New York: Springer-Verlag. ISBN 3-540-59179-6.

8. Parker, S. P (1988) Nuclear and Particle Physics Source Book. ISBN10:0070455090.

9. Zbiral, G (2012)"Does Gravitation Have an Influence on Electromagnetism?" Journal of Modern Physics 3: 1223 1230.

10. Williams, L. L (2012) "Electromagnetic Control of Space Time and Gravity: The Hard Problem of Interstellar Travel." Astronomical Review 7(2): 5.

11. Shifflett. J. A (2009) "A Modification of Einstein-Schrodinger Theory That Contains Einstein-Maxwell-YangMills Theory." Gen. Rel. Grav. 41: 1865-1886.

12. Lal, A. K (2011)“On Planetary Electromagnetism and Gravity." International Journal of Astronomy \& Astrophysics 1(2): 62-66. Doi:10.4236/ijaa.2011.12009.

13. Sweetser, D. B. "Unifying Gravity and Electromagnetism Using Analogies Based on Electromagnetism for Gravity." 1340 Commonwealth Ave. Apt. 7, Allston, MA 02134.

14. Wiese, U. -J (2009) Classical Field Theory. Institute for Theoretical Physics-Bern University.

15. Elyasi, N., and Boroojerdian, N (2011) "Affine Metrics and Algebroid Structures: Application to General Relativ- 
ity and Unification." International Journal of Theoretical Physics 51(10): 3160-3167.

16. Tiwari, S. C (2006) "Unified Field Theories and Einstein." Physics.

17. Marquet, P (2010) "Geodesics and Finslerian Equations in the EGR Theory." The Abraham Zelmanov Journal 3: 90-103.

18. Witten, L (1959) "Geometry of Gravitation and Electromagnetism.” Physical Review 115(1): 206-214.

19. Blau, M (2008) Lecture Notes on General Relativity. Albert Einstein Center for Fundamental Physics, Institute of Theoretical Physics, Bern University, CH-3012Bern, Switzerland.

20. Greiner, W (1980) Classical Electrodynamics. New York, Berlin, Heidelberg: Springer-Verlag.

21. Saha, G. B (2006) Physics and Radiobiology of Nuclear Medicine. New York: Springer.

22. Carey, V. P (1999) Statistical Thermodynamics and Microscale Thermophysics. Cambridge: Cambridge University Press, ISBN10: 0521652774.

23. Kompaneyets, A. S. Theoretical Physics: Volume 2. ASIN: B004SAS17M.

24. [24] Liboff, R. L. Introductory Quantum Mechanics. ISBN-13:9788131704417.

25. Gross, F. Relativistic Quantum Mechanics and Field Theory. ISBN-13:978-0471353867.

26. Biswas, S (2012) "Theory of Dynamic gravitational electromagnetism." Adv. Studies theor. Phys. 6(7): 339-354.

27. Boal, D. H. "Modern Physics from Quarks to Galaxies." Physics Department, Simon Fraser University

28. Sharif, M., and Bhatti, M. Z. U. H (2012) "Gravitational binding energy in charged cylindrical symmetry." Can. J. Phys. 20(90): 1-4.

29. Tsagas, C, G (2005) "Electromagnetic Fields in Curved Space Time." Class. Quantum Grav. 22: 393-407. DOI: 10.1088/0264-9381/22/2/011.

30. Clarkson, C. A., and Coley, A. A (2001) "Magnetic Fields and Cosmic Microwave Background.” 18: 1305. arxiv: astr-ph/0102172v2.

31. Carroll, S. M (1997) Lecture Notes on General Relativity. Arxiv: gr-qc/9712019v1.

32. Hung, G. Y (2004) "The Motion and Structure of Matter under Universal Magnetism.” Journal of Theoretics 6(6).

33. Ale'cian, E., and Morsink, S. M (2004) "The Effect of Neutron Star Gravitational Binding Energy on Radiation-Driven Mass-Transfer Binaries." The Astrophysical Journal 614: 914-921.

34. Carroll, S. "Does the Universe Need God?" Draft to appear in the Blackwell companion to science and Christianity.

35. Iliopoulos, I. A., and Tomaras, T. N (1985)“Gauge Invariance in Quantum Gravity." SLAC-PUB-3768, RU86/B/145.

36. Ellman, R (2004) “The Origin and Its Meaning." ISBN 978-1492100706

37. Macken, J. A. “The Universe Is Only Space Time." Santa Rose California; Original Draft_-February 2010; Revision 7.1-May 2013.
38. Rugh, S. E., and Zinkernagel, H (2002) "The Quantum Vacuum and the Cosmological Constant Problem." Studies in History \& Philosophy of Science Part B 33(4): $663-$ 705.

39. Staub, W. O. "Weyl's Theory of the Combined Gravitational-electromagnetic Field.” PhD Thesis, Pasadena, California.

40. Poplawski, N. J (2009) "Gravitation, Electromagnetism and the Cosmological Constant in Purely Affine Gravity. Foundations of Physics 39(3): 307-330.

41. Goenner, H. F. M (2004) "On the History of Unified Field Theories: Part 1." Living Rev. Relativity 7(2).

42. Ying, L. "Physical Null Conditions: Diameter of a Black HoleSingularity.”DOI:10.11648/j.ajmp.s.2015040101.18.

43. Weinberg, S (1989) "The Cosmological Constant Problem." Reviews of Modern Physics 61: 1.

44. Clark, C (2008) Quantum Electrodynamics. January 11, 2008.

45. Turro, N. J (2009) Principles of Molecular Photochemistry. University Science Books.

46. Sorli, A. 2014. "Gravity as a Result Quantum Vacuum Energy Density." In Proceedings of the IEEE International Conference on Electro/information Technology, 100-102.

47. Bondi, H., Pirani, F. A. E., and Robinson, I. "Gravitational Waves in General Relativity." Mathematical and Physical Sciences 251(1267): 519-533.

48. Tanner, P. A., Chua, M., and Reid, M. F (1993) "Energy Transfer by Magnetic Dipole-magnetic Dipole Interaction." Chemical Physics Letters 209(5): 6.

49. Peiris, H. V. The Homogeneous Universe. Department of Physics and Astronomy, University College London, Gower Street, London, WCIE6BT, U.K.

50. Saá, D (2009) Gravitation and Electromagnetism Unified. Storrs.

51. De Witt, B. S (1975) "Quantum Field Theory in Curved Space Time." Physics Reports 19(6): 295-357.

52. Weinberg, S. "Why Quantum Mechanics Might Need an Overhaul?" Science News.

Copyright: (C2021 Mohammed A. El-Lakany. This is an open-access article distributed under the terms of the Creative Commons Attribution License, which permits unrestricted use, distribution, and reproduction in anymedium, provided the original author and source are credited. 